\title{
A Chaotic Oscillator Based on Meminductor, Memcapacitor, and Memristor
}

\author{
Xingce Liu, Xiuguo Bi $\mathbb{D}$, Huizhen Yan, and Jun Mou \\ School of Information Science and Engineering, Dalian Polytechnic University, Dalian, 116034, China \\ Correspondence should be addressed to Xiuguo Bi; bixg@dlpu.edu.cn and Jun Mou; moujun@csu.edu.cn
}

Received 9 October 2021; Revised 17 November 2021; Accepted 20 November 2021; Published 23 December 2021

Academic Editor: Giacomo Innocenti

Copyright (c) 2021 Xingce Liu et al. This is an open access article distributed under the Creative Commons Attribution License, which permits unrestricted use, distribution, and reproduction in any medium, provided the original work is properly cited.

\begin{abstract}
In this paper, a hyperchaotic circuit consisting of a series memristor, meminductor, and memcapacitor is proposed. The dimensionless mathematical model of the system is established by the state equation of the circuit. The stability of equilibrium point of the system is analyzed by using the traditional dynamic analysis method. Then, the dynamical characteristics of the chaotic system with parameters are analyzed in detail. In addition, the system also has some particular phenomena such as attractor coexistence and state transition. Finally, the circuit is realized by DSP, and the result is consistent with that of numerical simulation. This proves the accuracy of the theoretical analysis. Numerical simulation result shows which hyperchaotic system has very abundant dynamical characteristics.
\end{abstract}

\section{Introduction}

The modern chaos theory was put forward by Henri Poincare, a famous French mathematical physicist, in the early nineteenth century. In 1903, to understand the stability of the Solar System, topological and dynamical systems were organically combined, in the process of which chaos in the conservative system was found [1]. In 1963, the Lorenz system was discovered by Lorenz, the father of chaos and a member of the American Academy of Sciences [2]. The Lorenz system is a common simplified model of atmospheric convective laser device, magneto-current generator, and several related convective problems. It is an important milestone significance as the starting point and cornerstone of chaos theory research for future generations. In 1971, the mathematician Takens F and physicist Ruelle D first used the concept of "chaos" to explain the mechanism of the emergence of turbulence, and the concept of "singular attractor" was first derived from dissipative systems [3]. In 1984, LO Chua proposed the Chua circuit, which further elaborated the chaos phenomenon in nonlinear circuits [4]. With the further study of chaos, researchers extend the study of chaos to fractional-order chaotic systems [5-13]. Of course, nonlinear circuits are still the focus of chaos research [14-16].
It is well known that nonlinear elements are an essential part of chaotic circuits. Memristor is the representative of nonlinear elements [17]. In 1971, Professor Chua proposed that there is a circuit element that can describe the relationship between magnetic flux value and charge value. He called this element memory resistance and theoretically proposed the existence of a fourth basic circuit element after resistance, inductance, and capacitance, called memristor [18]. In 1976, Professor Chua published a detailed description of the characteristics of memristor components, which laid a foundation for the research of memristors [19]. Since Professor Chua proposed the concept of memristors, no memristor has been found in practice for several decades. It was not until 2008, when HP Labs announced the first hardware implementation of a memristor device at the nanoscale, that research on the fourth type of device really took off $[20,21]$, and the research on memristors has made great progress [22-26]. Following the memristor, Professor Chua et al. proposed two special nonlinear elements in 2009, memcapacitor and meminductor [27], pointing out a new direction for the study of nonlinear chaotic circuits. Although Professor Chua has given the basic principles of memcapacitor and meminductor elements, due to technical means, material technology, and other reasons, so far there is 
no actual hardware memcapacitor and meminductor elements and few theoretical analysis and research on memcapacitor and meminductor elements. However, based on the basic principle given by Professor Chua, the research on memcapacitor and meminductor circuit is gradually deepening [28-34].

By replacing Chua's diode with a memristor, a chaotic system was constructed for the first time in 2008 [35], and detailed theoretical analysis and numerical simulation of the circuit were carried out. In 2012, Professor Chua and Bharathwaj Muthuswamy formally proposed a minimalist chaotic circuit based on a memristor, which consists of only one memristor, one inductor, and one capacitor [36]. In 2013, Professor Xu improved the minimalist memristor system and proposed a minimalist parallel memristor circuit that combines a memristor, a capacitor, and an inductor in parallel [37]. In 2019, Fang Yuan made the simplest parallel system by connecting the memristor, meminductor, and memcapacitor in parallel and simulated it [38]. The chaotic sequence generated by the chaotic circuit based on memory element also has good pseudorandomness, which makes it have wider application prospect in image encryption, chaotic secure communication, and neural network [39-58].

However, no serial circuit of meminductor, memcapacitor, and memristor has been reported. A chaotic oscillator composed of memcapacitor, meminductor, and memristor in series is presented in this paper. The advantages of the system are that, different from general chaotic circuits, the dynamic behavior of chaotic circuits will be more abundant due to the basic characteristics of meminductor, memcapacitor, and memristor [59-62]. Compared with other simplest circuits, the system constructed in this paper can generate more pseudorandom sequences and can be better used in chaotic encryption. Moreover, the system has the phenomenon of hyperchaos, which is not found in the previous simplest series circuit. The disadvantage of this system is that the phase diagram of chaotic attractor is too simple and no new chaotic attractor is generated. Finally, we complete the dynamic analysis of the chaotic system and implement the chaotic circuit by DSP, which proves the realizability of the chaotic circuit. Compared with analog circuits, digital circuits have higher accuracy and are less susceptible to interference from the external environment [63-65]. Therefore, the digital circuit is more suitable for the realization of this circuit.

The rest of the article reads as follows: the models of meminductor, memcapacitor, and memristor are introduced in Section 2. The first-order differential equations of the chaotic system are constructed according to the chaotic circuit, and the mathematical model is obtained by dimensionless processing. The stability of equilibrium point and the dynamic behaviors of chaotic system are analyzed by traditional methods in Section 3. We introduce how to realize the circuit on DSP and the result of realization and compares with the result of numerical simulation in Section 4. The conclusion of this study is drawn in Section 5.

\section{Models of Meminductor, Memristor, and Memcapacitor}

2.1. Model of the Memristor. According to Chua's description, the memristor can be defined as

$$
\left\{\begin{array}{l}
x=g(y, z, t) z \\
\dot{y}=f(y, z, t)
\end{array}\right.
$$

where $x$ and $z$ are the output and input of the memristor and $y$ is the internal state variable of the memristor.

A charge-controlled memristor proposed by Professor Chua is used for reference in this paper.

$$
\left\{\begin{array}{c}
V_{M}=a\left(y^{2}-1\right) i_{M} \\
\dot{y}=i_{M}-b y-i_{M} y
\end{array},\right.
$$

where $V_{\mathrm{M}}$ and $i_{\mathrm{M}}$ are the voltage and current passing through the memristor, respectively, $y$ is the internal state variable of the memristor, and $b$ and $a$ are the internal parameters of the memristor.

For the charge-controlled memristor defined in formula (2), if a sinusoidal current source with an effective value of $10 \mathrm{~A}$ and a frequency of $10 \mathrm{~Hz}$ is added at both ends, its $v-i$ characteristic curve is shown in Figure 1(a). The curve is a compact-like hysteresis loop shaped like an italic 8. Except at the origin, the current and voltage are double-valued functions of each other. With the increase in frequency, the side lobe area of hysteresis curve decreases, which is consistent with the characteristics described by Chua's and HP's memristors.

2.2. Model of the Meminductor. The meminductor element is a typical two-terminal passive element, like flux-controlled memristor and charge-controlled memristor element. For a typical smooth meminductor element, the magnetic flux gathered at both ends of the meminductor element is defined as $\Phi$, the current passing through the meminductor element is defined as $i$, and the time integral of the current $i$ is defined as $q$. At this point, the state variable of the meminductor element can be defined as

$$
\left\{\begin{array}{l}
i(t)=L_{M}^{-1}(\rho) \phi(t), \\
L_{M}^{-1}(\rho)=c+d \rho .
\end{array}\right.
$$

The $\rho$ represents the integral when the flux $\Phi$ through the meminductor, and $c$ and $d$ are the parameters of the meminductor:

$$
\rho=\int_{t_{0}}^{t} \phi(\tau) d \tau .
$$

For the meminductor element, if the magnetic flux gathered at both ends is a sinusoidal quantity with a size of $2 \mathrm{~Wb}$ and a frequency of $1 \mathrm{~Hz}$, then the characteristic curve of $\Phi-i$ on the smooth memory container element is as shown in Figure 2(a). It can be seen from Figure 2 that the 


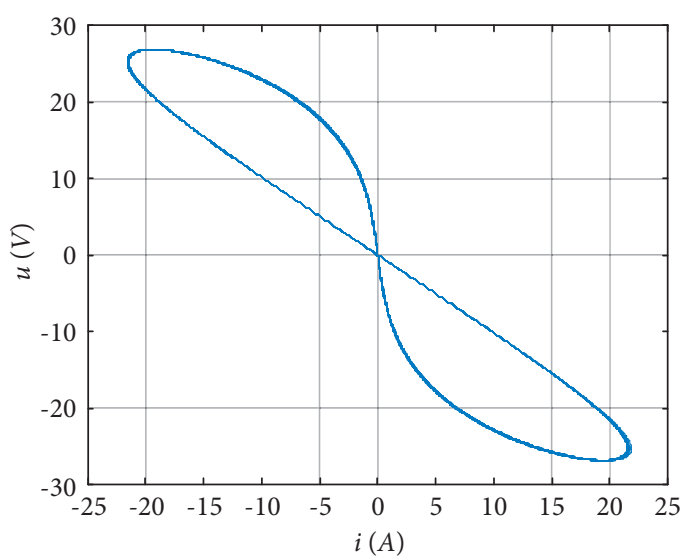

(a)

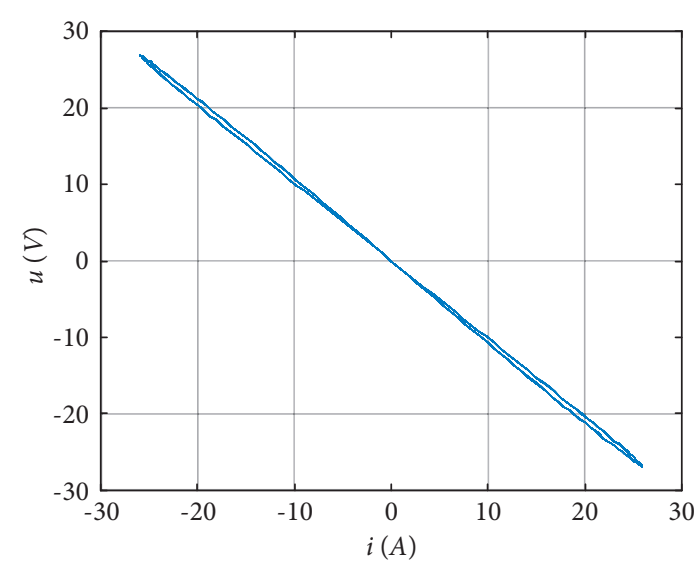

(b)

FIgURE 1: $v-i$ characteristics curve of the memristor with $a=1.7$ and $b=1$. (a) $f=10 \mathrm{~Hz}$. (b) $f=50 \mathrm{~Hz}$.

$\Phi-i$ characteristic curve similar to the italic " 8 " type can be obtained in all meminductor elements. With the increase of frequency, the side lobe area of hysteresis curve decreases, which is consistent with the characteristics of the meminductor element.

2.3. Model of the Memcapacitor. According to the basic characteristics of memcapacitor, the definition of ideal charge-controlled memcapacitor could be obtained as follows:

$$
\left\{\begin{array}{l}
u(t)=C_{M}^{-1}(\sigma) q(t), \\
C_{M}^{-1}(\sigma)=e+g \sigma .
\end{array}\right.
$$

The $u(t)$ and $q(t)$ represent the voltage through the memcapacitor and the charge, $\sigma$ represents the integral when the flux $q$ through the memcapacitor, and e and $g$ are the parameters of the memcapacitor.

$$
\sigma=\int_{t_{0}}^{t} q(\tau) d \tau
$$

For the memcapacitor defined in formula (6), if sinusoidal AC power supply is added at both ends, its Volt-Coulomb characteristic curve is as shown in Figure 3. Its curve is an inclined 8; shaped hysteresis loop shape, and its side lobe area gradually decreases with the increase in frequency, which conforms to the basic characteristics of the memcapacitor.

\section{A New Simple Chaotic Oscillator}

3.1. A New Chaotic Oscillator. According to the basic characteristics of meminductor, memristor, and memcapacitor introduced in the previous section, a new chaotic circuit of memristor, meminductor, and memcapacitor in series is designed on the basis of the simplest series circuit. The circuit is shown in Figure 4.

The circuit is composed of memristor, meminductor, and memcapacitor in series. According to the circuit, the first-order differential equations of the circuit can be obtained. Here, $\Phi$ is the magnetic flux of the meminductor, $y$ is the state variable of the memristor, $q$ represents the amount of charge accumulated at both ends of the memcapacitor, the time integral of $q$ is defined as $\sigma$, and the time integral of $\Phi$ is defined as $\rho$.

$$
\left\{\begin{array}{l}
\dot{\phi}=-u_{c}-u_{M} \\
\dot{y}=i-d y-i y \\
\dot{q}=i \\
\dot{\sigma}=q \\
\dot{\rho}=\phi .
\end{array}\right.
$$

Let $\Phi=x, y=y, q=z, \sigma=u$, and $\rho=w$; employing the normalized operation, then the dimensionless equations can be expressed.

$$
\left\{\begin{array}{l}
\dot{x}=-(c+d u) z-a\left(y^{2}-1\right)(e+g w) x \\
\dot{y}=(e+d w) x-b y-(e+g w) x y \\
\dot{z}=(e+g w) x \\
\dot{u}=z \\
\dot{w}=x .
\end{array}\right.
$$

For the parameters of the system as $a=30, b=18, d=2$, $c=100, e=0.2$, and $g=-0.017$, the initial conditions are $(4$, $50,1,24,-23)$. The chaotic attractor of the system obtained by numerical simulation with MATLAB is shown in Figure 5 .

3.2. Equilibrium Point Set and Stability. The divergence of the system can be expressed as

$$
\begin{aligned}
\nabla V & =\frac{\partial \dot{x}}{\partial x}+\frac{\partial \dot{y}}{\partial y}+\frac{\partial \dot{z}}{\partial z}+\frac{\partial \dot{u}}{\partial u}+\frac{\partial \dot{w}}{\partial w} \\
& =-a\left(y^{2}-1\right)(e+g w)-b-(e+g w) x
\end{aligned}
$$

The parameters of the system are $a=30, b=18, d=2$, $c=100, e=0.2$, and $g=-0.017$, and the initial conditions are 


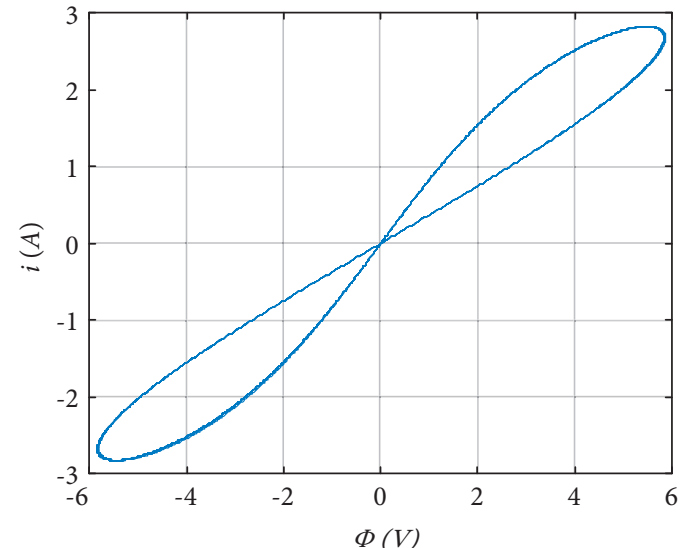

(a)

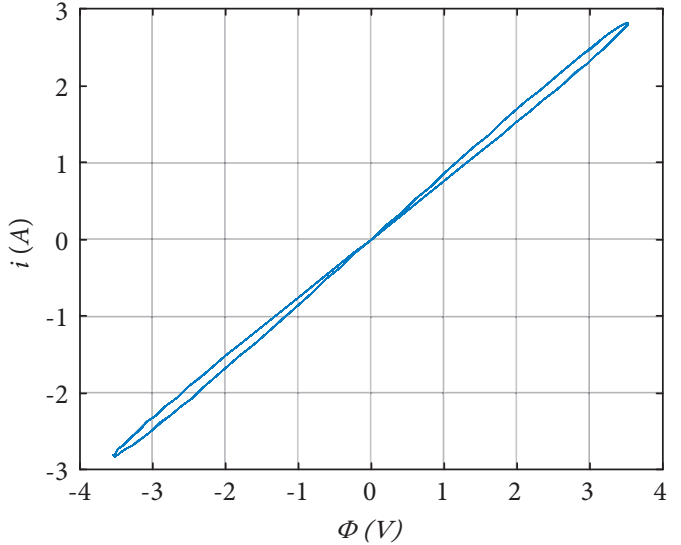

(b)

Figure 2: $\Phi$-i curve of the meminductor. (a) $c=1, d=1$, and $f=1 \mathrm{~Hz}$. (b) $c=1, d=1$, and $f=10 \mathrm{~Hz}$.

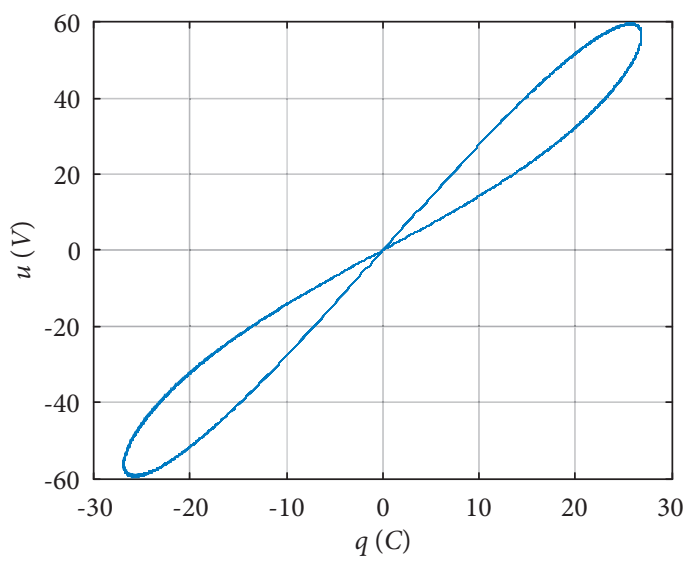

(a)

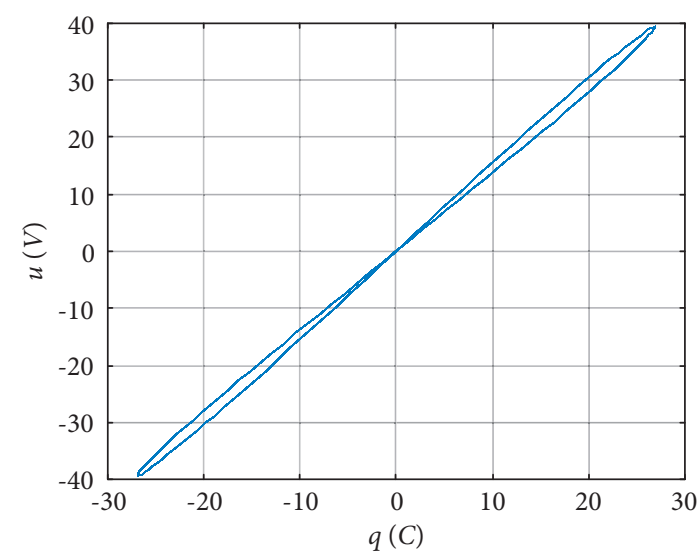

(b)

Figure 3: Hysteresis curve of the memcapacitor. (a) $e=1, g=1$, and $f=10 \mathrm{~Hz}$. (b) $e=1, g=1$, and $f=80 \mathrm{~Hz}$.

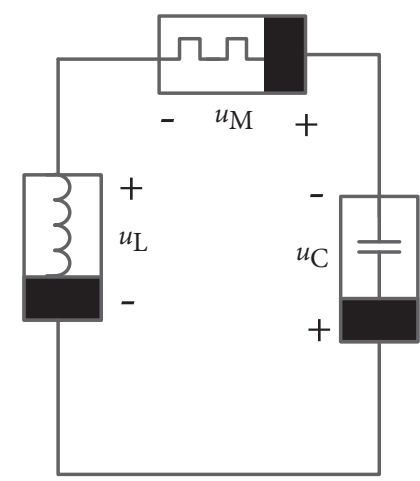

Figure 4: A novel memristive chaotic circuit. 


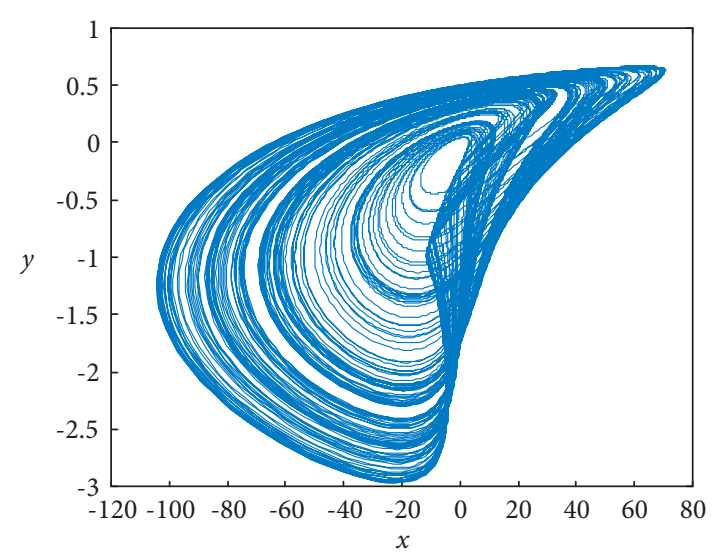

(a)

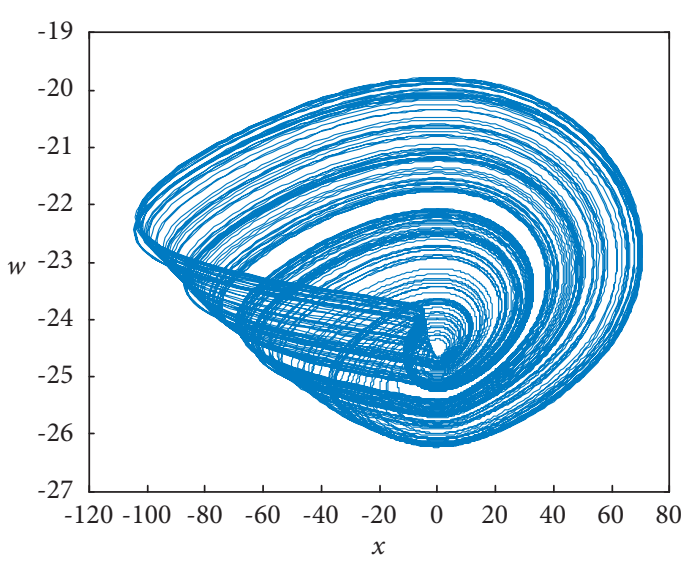

(b)

FIgURE 5: Phase diagram of the system. (a) $x-y$ plane. (b) $x-w$ plane.

$(4,50,1,24,-23)$. The divergence of (9) is -44343 , and it is less than zero. This means that it is a dissipative system, and the system might be a chaotic system.

Solving the equations $\dot{x}=\dot{y}=\dot{z}=\dot{u}=\dot{w}=0$, we get the equations of the system as

$$
\left\{\begin{array}{l}
-(c+d u) z-a\left(y^{2}-1\right)(e+g w) x=0 \\
(e+g w) x-b y-(e+g w) x y=0 \\
(e+g w) x=0 \\
z=0 \\
x=0
\end{array}\right.
$$

The equilibrium point set of (8) can be set as $Q(0,0,0, n$, $m$ ), which means the system has infinite equilibria. According to (8), the Jacobian matrix $J_{\mathrm{E}}$ of the system can be expressed as

$$
\begin{aligned}
J_{E} & =\left[\begin{array}{ccccc}
-a\left(y^{2}-1\right)(e+g w) & -2 a y(e+g w) x & -(c+d u) & -d z & -a g\left(y^{2}-1\right) x \\
(e+g w)-(e+g w) y & -b-(e+g w) x & 0 & 0 & g x-g x y \\
(e+g w) & 0 & 0 & 0 & g x \\
0 & 0 & 1 & 0 & 0 \\
1 & 0 & 0 & 0 & 0
\end{array}\right] \\
& =\left[\begin{array}{ccccc}
a(e+g m) & 0 & -(c+d n) & 0 & 0 \\
(e+g m) & -b & 0 & 0 & 0 \\
(e+g m) & 0 & 0 & 0 & 0 \\
0 & 0 & 1 & 0 & 0 \\
1 & 0 & 0 & 0 & 0
\end{array}\right] .
\end{aligned}
$$

Then, we can get the characteristic polynomial of the equilibrium point set $Q$ is

$$
\lambda^{2}\left(\lambda^{3}+m_{1} \lambda^{2}+m_{2} \lambda+m_{3}\right)=0,
$$

where $m_{1}=b-a(e+g m), m_{2}=[(e+g m)(c+d n)-a b(e+g m)]$, and $m_{3}=b((e+g m)(c+d n)$.

(10) means system (4) has three nonzero eigenvalues and one zero eigenvalue, where $m_{1}, m_{2}$, and $m_{3}$ are coefficients. With the Routh-Hurwitz stable theorem, system (4) is stable if $m_{1}>0, m_{1} m_{2}-m_{3}>0$, and $m_{3}\left(m_{1} m_{2}-m_{3}\right)>0$. The chaotic state of the system requires that at least one eigenvalue must be positive, so $m_{1}, m_{1} m_{2} m_{3}$, and $m_{3}$ should not be all positive. Hence, we could set $Q$ such as $Q_{1}(0,0,0,10,20)$; for the parameters of the system as $a=30, b=18, d=2, c=100$, $e=0.2$, and $g=-0.017$, we can obtain $m_{1}=1.8>0, m_{2}=$ $226.8>0, \quad m_{1}=1166.4>0, \quad m_{1} m_{2}-m_{3}<0, \quad m_{3}\left(m_{1} m_{2}-m_{3}\right)$ $<0$, and $\lambda_{1}=\lambda_{2}=0, \lambda_{3}=-18, \lambda_{4}=9$, and $\lambda_{5}=7.2$, which means that the system is unstable and has the potential to produce chaos. 
3.3. Impact of the Parameters. When the system parameters are $d=2, b=18, c=100, e=0.2$, and $g=-0.017$, the initial system conditions are $(4,50,1,24,-23)$, and only parameter $a$ is changed, the bifurcation diagram and its corresponding Lyapunov exponents can be calculated when parameter $a$ increases from 10 to 60, as shown in Figure 6.

Through the analysis of Lyapunov exponents, it is not difficult to find that there are two positive Lyapunov exponents in the system, which indicates the existence of hyperchaos in the system. By observing the bifurcation diagram, we can know that with the further increase in circuit parameter $a$, the system enters into chaos state through the period-doubling bifurcation process. It is found that the LEs and the bifurcation diagram are comparable. The system is bisected by antiperiodic doubling. The changes of each state with parameter $a$ are shown in Table 1 .

In order to understand the dynamical behavior of the simplest memristor chaotic system, the parameters are set to $a=30, d=2, c=100, e=0.2$, and $g=-0.017$, the initial system conditions are $(4,50,1,24,-23)$, and the internal variable $b$ of the memristor in the system is set to be variable, where $b \in[1,55]$. In this way, Lyapunov exponents and bifurcation diagram of the integer-order minimalist memristor chaotic system with variable $b$ can be obtained, as shown in Figure 7.

In order to better express the dynamic characteristics of the system, when the internal parameter $b$ of the memristor changes, the complex dynamical behaviors of the chaotic circuit of the integer-order minimalist memristor are as summarized in Table 2.

With the parameters of the system as $b=18, a=30$, $c=100, e=0.2$, and $g=-0.017$ and setting the initial condition as $(4,50,1,24,-23)$, when the parameter $d$ increases, LEs and bifurcation diagram of the integer-order minimalist memristor chaotic system with variable $d$ can be obtained, as shown in Figure 8. It can be seen from the observation that the Lyapunov exponents are basically consistent with the bifurcation diagram.

According to LEs, when parameter $d$ changes, the state of the system changes very frequently and there is the hyperchaos phenomenon. It can be seen from the bifurcation diagram that the system produces chaos through inverse period doubling. Bifurcation diagram and Lyapunov exponents are in good agreement. With the change of parameter $d$, the dynamic state of chaotic system is detailed in Table 3.

Let the system parameters be $b=18, a=30, c=100, d=2$, and $g=-0.017$, the initial values of system $(4,50,1,24,-23)$ remain unchanged, and only change the system parameter $e$. The Lyapunov exponential spectrum of parameter $E$ and its corresponding bifurcation diagram can be obtained through calculation, and the result is shown in Figure 9.

In order to more intuitively display the dynamic behaviors of the system as parameter $e$ changes, we made Table 4 to show the system state in detail as parameter $e$ changes, and the results are shown.

3.4. LE Dimension. LE dimension is also an important method to judge whether the system is in chaos state. In general, directions with positive and zero Lyapunov exponents all contribute to the supporting attractor, while the negative Lyapunov exponents correspond to the contraction direction and contribute to the fractional part of the attractor dimension after counteracting the effect of expansion direction. Rank all Lyapunov exponents from highest to lowest:

$$
\sigma_{1} \geq \sigma_{2} \geq \sigma_{3} \geq \ldots \geq \sigma_{i} \geq \ldots
$$

Then, start from the largest $\sigma$ (at least one of the chaotic motion exponents is greater than zero), add the subsequent exponents one by one, and when it adds to $\sigma_{k}$, the sum $S_{k}$ is nonnegative, and the sum $S_{k+1}$ is negative when added to the next 0 , so it is natural to assume that the attractor dimension is between $k$ and $k+1$. Therefore, LE dimension of the attractor is defined as

$$
D_{L}=\frac{k+S_{k}}{\sigma_{k+1}} .
$$

According to the above calculation method, the parameters are $c=100$ and $g=-0.017$ and the initial value of the system is $(4,50,1,24,-23)$. We can get the LE dimension diagram of parameters $a, b, d$, and $e$ as shown in Figure 10.

3.5. Complexity Analysis. For the chaotic systems, the most valuable research object is chaotic interval. When the system is in the chaotic state, the pseudorandomness of the sequence generated by it is the highest, which indicates that it has the best decoding resistance. The greater the complexity value of the chaotic system, the higher the degree of chaos, the stronger the pseudorandomness of the generated sequence, and the more reliable the system is. Therefore, in the field of chaotic secure communication, the parameter range with high complexity is often the best range for key selection. In order to learn the dynamical characteristics of chaotic system, SE (spectral entropy) and C0 complexity algorithms are used to calculate and analyze the system. Set the initial values $(4,50,1,24,-23)$, and keep other parameters unchanged. Set parameters $a \in[10,60]$ and $d \in[0.1,6]$; as parameters $a$ and $d$ change, the complexity of $\mathrm{C} 0$ and SE is as shown in Figure 11(a) and 11(b). The value of system complexity in the diagram is very obvious. By analyzing its complexity value, we can understand the state change of the chaotic system. When $a=55.17$ and $d=3.07$, the complexity value of the system is high, presenting chaos state. When $a=11.33$ and $d=3.05$, the complexity value of the system is relatively low and presents a periodic window. Although SE and $\mathrm{C} 0$ have different complexity values, they tend to change in the same way, because they are all based on FFT algorithms.

By analyzing the complexity, the state of chaotic attractor can be understood more intuitively; when $a=55.17$ and $d=3.07$, the system is chaotic. When $a=11.33$ and $d=3.05$, the system is periodic. The result is shown in Figure 12.

3.6. State Transition. If the system evolves from different initial domains, special chaotic dynamics characteristics, namely, state transition and transient chaos [66], will appear. The system in this paper has the phenomenon of state 


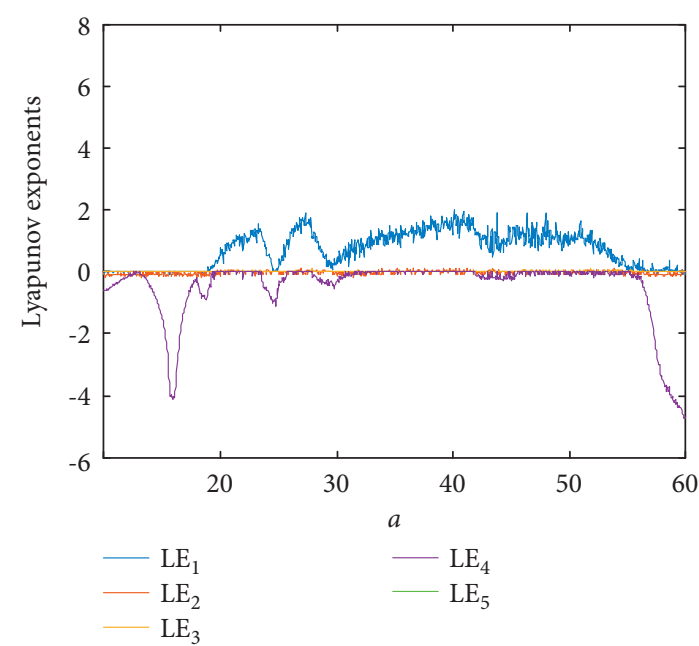

(a)

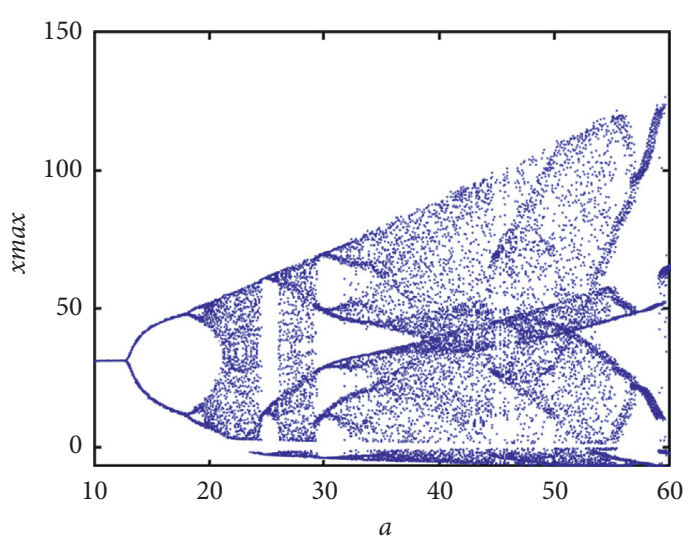

(b)

Figure 6: (a) LE spectrum and (b) bifurcation diagram with parameter $a$.

TABLE 1: The states and LEs corresponding to parameter $a$.

\begin{tabular}{|c|c|c|}
\hline Range & LEs & State \\
\hline $10-19$ & $0 \ldots$ & Period \\
\hline $19-24.5$ & $+0--$ & Chaos \\
\hline $24.5-24.7$ & $0 \ldots$ & Period \\
\hline $24.7-29.5$ & $+0--$ & Chaos \\
\hline $29.5-29.7$ & $0 \ldots$ & Period \\
\hline $29.7-33.8$ & $+0--$ & Chaos \\
\hline $33.8-34$ & $++0--$ & Hyperchaos \\
\hline $34-35.1$ & $+0--$ & Chaos \\
\hline $35.1-35.3$ & $++0--$ & Hyperchaos \\
\hline $35.3-36$ & $+0--$ & Chaos \\
\hline $36-36.3$ & $++0--$ & Hyperchaos \\
\hline $36.3-36.5$ & $+0--$ & Chaos \\
\hline $36.5-36.7$ & $++0--$ & Hyperchaos \\
\hline $36.7-37.5$ & $+0-$ & Chaos \\
\hline $37.5-37.7$ & $++0--$ & Hyperchaos \\
\hline $37.7-39.1$ & $+0--$ & Chaos \\
\hline $39.1-39.3$ & $++0--$ & Hyperchaos \\
\hline $39.3-40.1$ & $+0--$ & Chaos \\
\hline $40.1-40.3$ & $++0--$ & Hyperchaos \\
\hline $40.3-41.1$ & $+0--$ & Chaos \\
\hline $41.1-41.3$ & $++0--$ & Hyperchaos \\
\hline $41.3-55.1$ & $+0--$ & Chaos \\
\hline $55.1-55.3$ & $0 \ldots$ & Period \\
\hline $55.3-56.4$ & $+0--$ & Chaos \\
\hline $56.4-58.2$ & $0 \ldots$ & Period \\
\hline $58.2-58.8$ & $+0--$ & Chaos \\
\hline $58.8-59.5$ & $+0--$ & Weak chaos \\
\hline $59.5-60$ & $0 \ldots$ & Period \\
\hline
\end{tabular}

transition. For the parameters of the system with $a=30$, $b=18, d=2, c=100, e=0.2$, and $g=-0.017$, the initial conditions are $(4,50,1,24,-23)$. The timing diagram of the chaotic system of the first sequence with $t=[0,1000]$ is shown in Figure 13.

After $116 \mathrm{~s}$, the system changes from the periodic state to chaotic state. When $t=[116,480]$, the system is in the chaotic state; after that, the system enters the periodic state; when $t=[480,497]$, the system is in the periodic state. Then, the system enters the chaos state. When $t=[497,836]$, the system is in the chaos state, and finally, the system enters the periodic state. When $t=[480,497]$ and $t=[497,836]$, the sequence diagram of the system is as shown in Figure 14. 


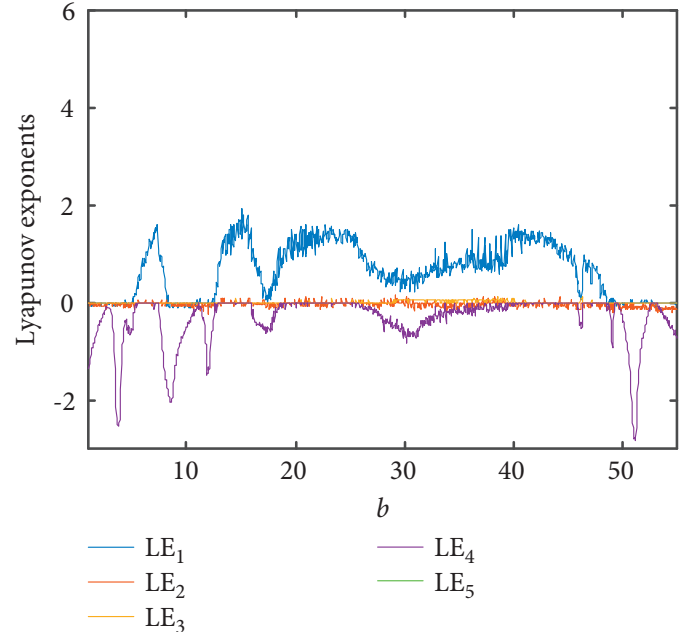

(a)

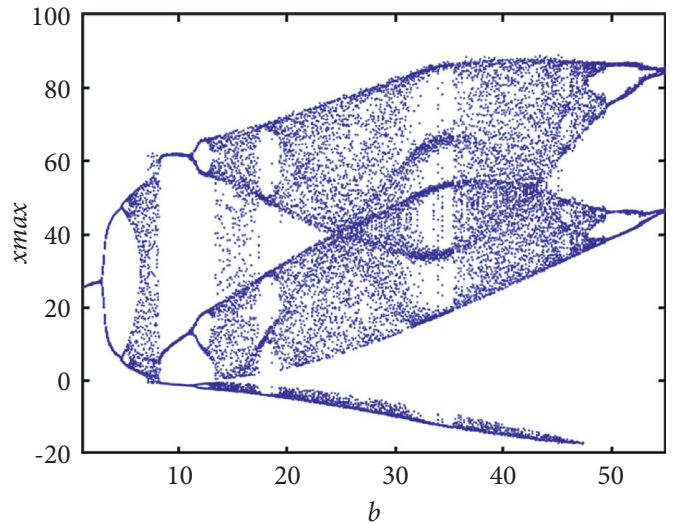

(b)

FIgURE 7: (a) LEs spectrum and (b) bifurcation diagram with parameter $b$.

TABLE 2: The states and LEs corresponding to different parameter $b$ values.

\begin{tabular}{|c|c|c|}
\hline Range & LEs & State \\
\hline $1-5$ & $0 \ldots$ & Period \\
\hline $5-8.2$ & $+0--$ & Chaos \\
\hline $8.2-12.5$ & $0 \ldots$ & Period \\
\hline $12.5-17$ & $+0---$ & Chaos \\
\hline $17-17.4$ & $0 \ldots$ & Period \\
\hline $17.4-20.8$ & $+0--$ & Chaos \\
\hline $20.8-21.1$ & $++0--$ & Hyperchaos \\
\hline $21.1-38.5$ & $+0--$ & Chaos \\
\hline $38.5-39.5$ & $++0-$ & Hyperchaos \\
\hline $39.5-46.1$ & $+0--$ & Chaos \\
\hline $46.1-46.3$ & $0 \ldots$ & Period \\
\hline $46.3-48.7$ & $+0--$ & Chaos \\
\hline 48.7-55 & $0 \ldots$ & Period \\
\hline
\end{tabular}

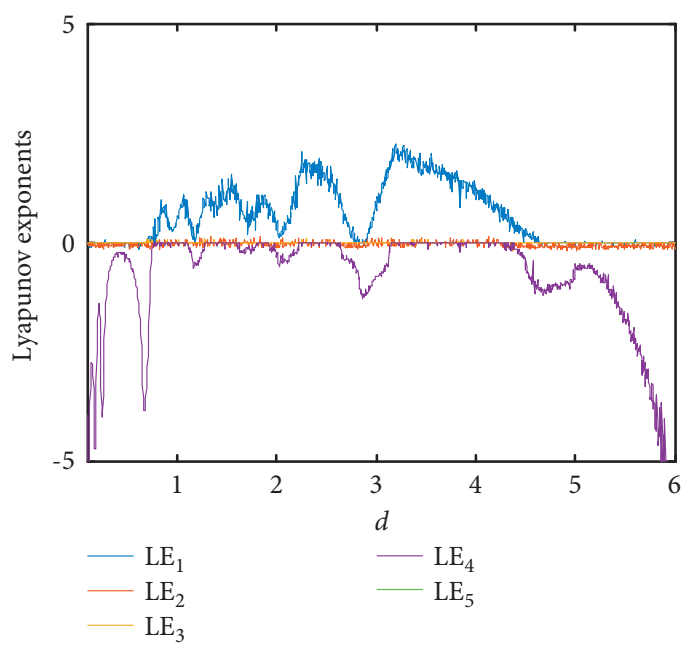

(a)

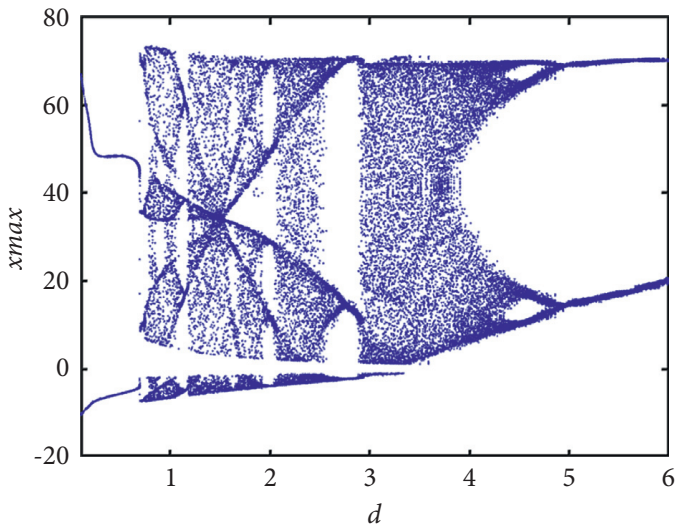

(b)

Figure 8: (a) LEs spectrum and (b) bifurcation diagram with parameter $d$. 
TABLE 3: The states and LEs corresponding to different parameter $d$ values.

\begin{tabular}{|c|c|c|}
\hline Range & LEs & State \\
\hline $0.1-0.68$ & $0 \ldots$ & Period \\
\hline $0.68-1.17$ & $+0--$ & Chaos \\
\hline $1.17-1.18$ & $0 \ldots$ & Period \\
\hline $1.18-1.46$ & $+0--$ & Chaos \\
\hline $1.46-1.48$ & $++0--$ & Hyperchaos \\
\hline $1.48-1.76$ & $+0--$ & Chaos \\
\hline $1.76-1.78$ & $++0-$ & Hyperchaos \\
\hline $1.78-2.05$ & $+0--$ & Chaos \\
\hline $2.05-2.07$ & $++0--$ & Hyperchaos \\
\hline $2.07-2.79$ & $+0--$ & Chaos \\
\hline $2.79-2.91$ & $0 \ldots$ & Period \\
\hline $2.91-4.12$ & $+0--$ & Chaos \\
\hline $4.12-4.14$ & $++0-$ & Hyperchaos \\
\hline $4.14-4.65$ & $+0--$ & Chaos \\
\hline $4.65-6$ & $0 \ldots$ & Period \\
\hline
\end{tabular}

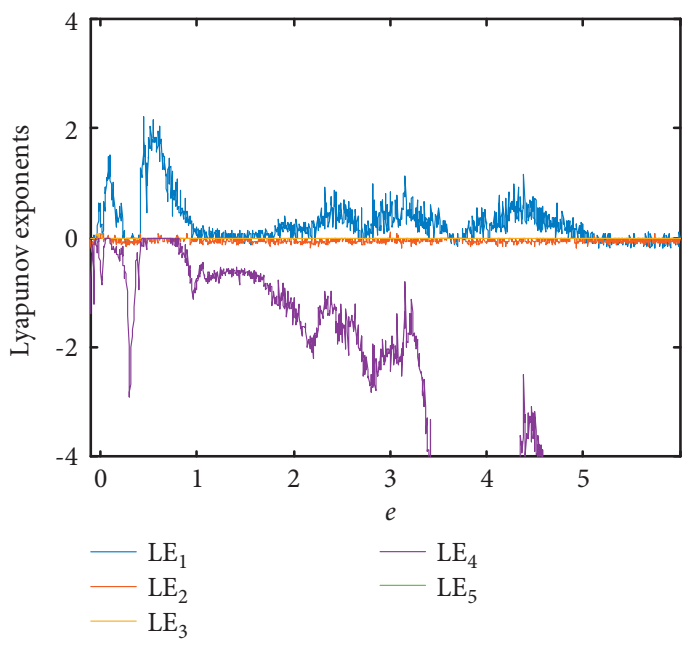

(a)

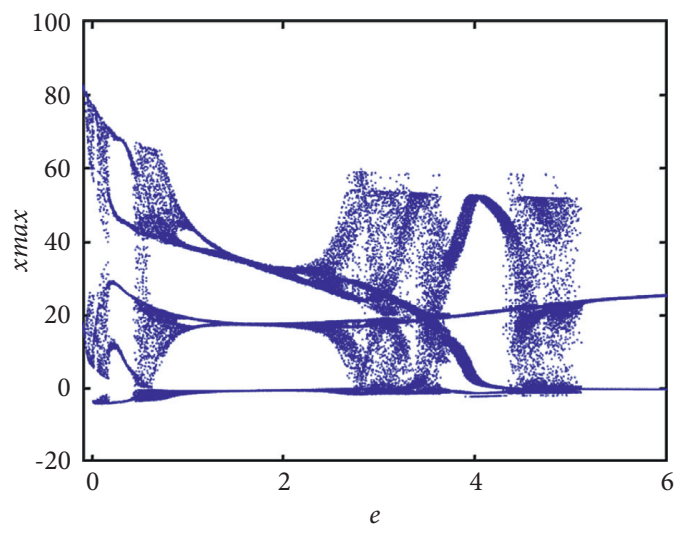

(b)

FIGURE 9: (a) LEs spectrum and (b) bifurcation diagram with parameter $e$.

When $t=[480,497]$ and $t=[497,836]$, the chaotic attractor phase diagram of the system is as shown in Figure 15.

3.7. Coexistence of Attractors. Coexistence of attractors is a peculiar phenomenon in chaotic systems, which is widely used in chaotic image encryption [67-70]. When the system parameters remain unchanged and only the initial state of the system is changed, the motion orbit of the system will gradually tend to different states. In order to understand the particular phenomenon, let $a=30, b=18, d=2, c=100$, $e=0.2$, and $g=-0.017$ and the initial values be $\left(4,50, z_{0}, 24\right.$, $w_{0}$ ), with the initial values $z_{0}$ and $w_{0}$ varying; the changes in the position and shape of the chaotic attractor can be found. The results are shown in Figure 16.

Let $z_{0}=1,10,20,30,40$, and 50 . The coexistence of the six chaotic attractors in the $w-x$ plane is shown in Figure 16(a), where the blue, red, yellow, purple, green, and light blue attractors express the size and position with the initial conditions of $(4,50,1,24,-23),(4,50,10,24,-23),(4,50,20$, $24,-23),(4,50,30,24,-23),(4,50,40,24,-23)$, and $(4,50$, $50,24,-23)$, respectively.

When $w_{0}=-10,-20,-30,-40,-50$, and -60 , the coexistence of the six chaotic attractors in the $w-x$ plane is shown in Figure 16(b), where the blue, red, yellow, purple, green and light blue attractors express the size and position with the initial conditions of $(4,50,1,24,-60),(4,50,1$, $24,-50),(4,50,1,24,-40),(4,50,1,24,-30),(4,50,1,24$, $-20)$, and $(4,50,1,24,-10)$, respectively.

\section{DSP Implementation}

DSP is digital signal processing technology. Digital signal processing is the use of computer or special processing equipment, in digital form of signal acquisition, transformation, filtering, valuation, enhancement, compression, 
TABLE 4: The states and LEs corresponding to different parameter $e$ values.

\begin{tabular}{|c|c|c|}
\hline Range & LEs & State \\
\hline$-0.1 \sim-0.05$ & $0 \ldots$ & Period \\
\hline$-0.05-0.255$ & $+0--$ & Chaos \\
\hline $0.255-0.32$ & $0 \ldots$ & Period \\
\hline $0.32-0.34$ & $+0--$ & Chaos \\
\hline $0.34-0.41$ & $0 \ldots$ & Period \\
\hline $0.41-1.085$ & $+0--$ & Chaos \\
\hline $1.085-1.09$ & $0 \ldots$ & Period \\
\hline $1.09-1.11$ & $+0--$ & Chaos \\
\hline $1.11-1.12$ & $0-\cdots$ & Period \\
\hline $1.12-1.24$ & $+0--$ & Chaos \\
\hline $1.24-1.29$ & $0 \ldots$ & Period \\
\hline $1.29-1.305$ & $+0--$ & Chaos \\
\hline $1.305-1.515$ & $0 \ldots$ & Period \\
\hline $1.515-1.555$ & $+0--$ & Chaos \\
\hline $1.555-1.565$ & $0 \ldots$ & Period \\
\hline $1.565-1.645$ & $+0--$ & Chaos \\
\hline $1.645-1.655$ & $0 \ldots$ & Period \\
\hline $1.655-1.685$ & $+0--$ & Chaos \\
\hline $1.685-1.695$ & $0 \ldots$ & Period \\
\hline $1.695-1.785$ & $+0--$ & Chaos \\
\hline $1.785-1.795$ & $0 \ldots$ & Period \\
\hline $1.795-2.685$ & $+0--$ & Chaos \\
\hline $2.685-2.695$ & $0 \ldots$ & Period \\
\hline $2.695-2.72$ & $+0--$ & Chaos \\
\hline $2.72-2.73$ & $0 \ldots$ & Period \\
\hline $2.73-3.66$ & $+0---$ & Chaos \\
\hline $3.66-3.745$ & $0 \ldots$ & Period \\
\hline $3.745-3.785$ & $+0--$ & Chaos \\
\hline $3.785-3.79$ & $0 \ldots$ & Period \\
\hline $3.79-5.055$ & $+0--$ & Chaos \\
\hline $5.055-6$ & $0 \ldots$ & Period \\
\hline
\end{tabular}

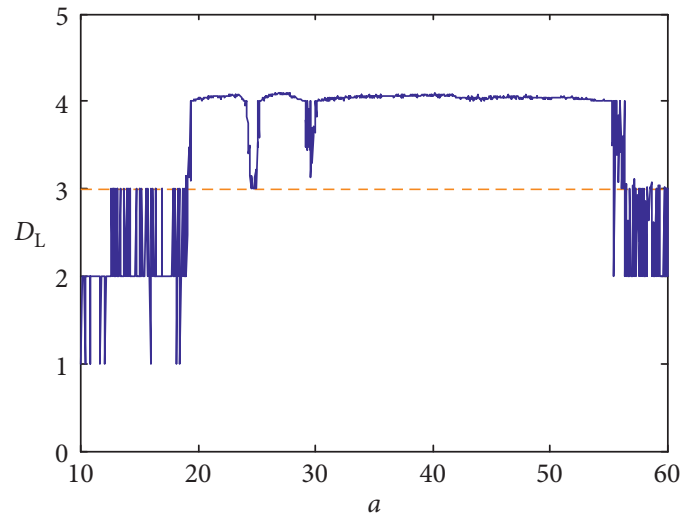

(a)

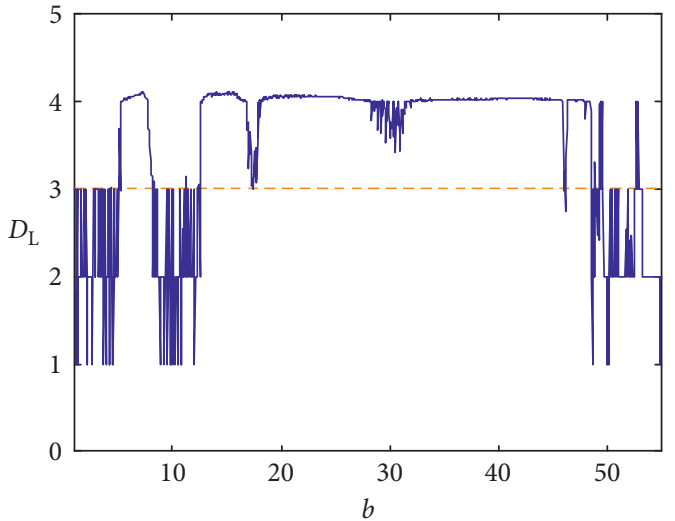

(b)

Figure 10: Continued. 


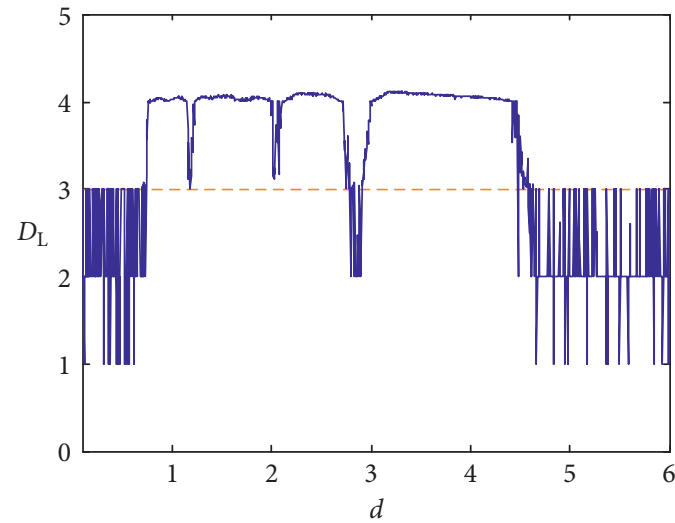

(c)

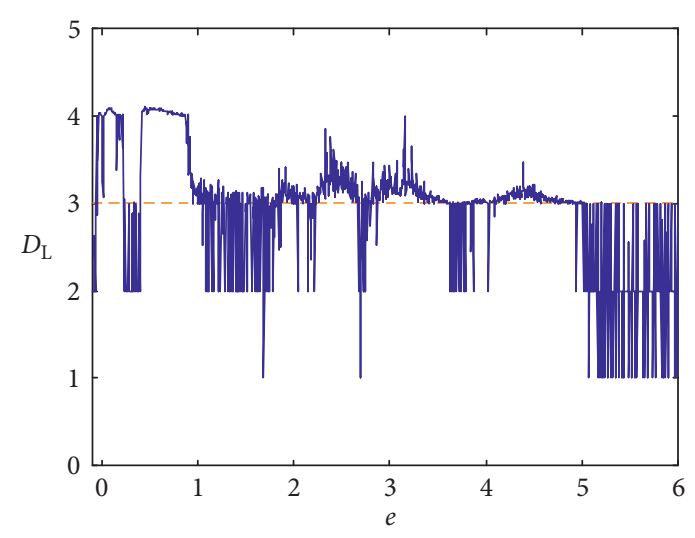

(d)

FIgURE 10: LE dimension. (a) $a \in(10,60)$, (b) $b \in(1,55)$, (c) $d \in(0.1,6)$, and (d) $e \in(-0.1,6)$

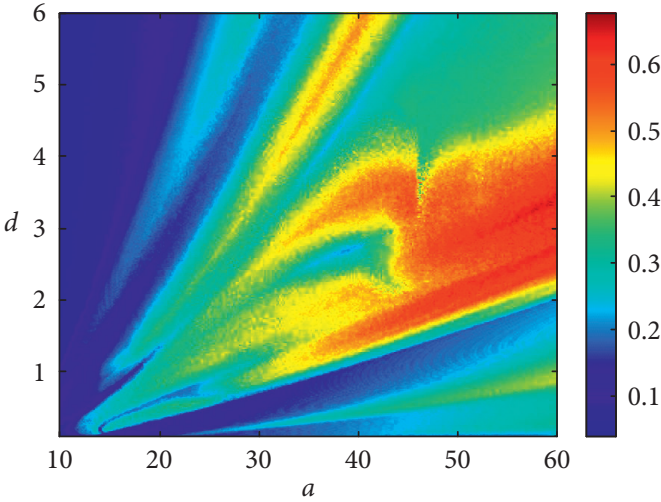

(a)

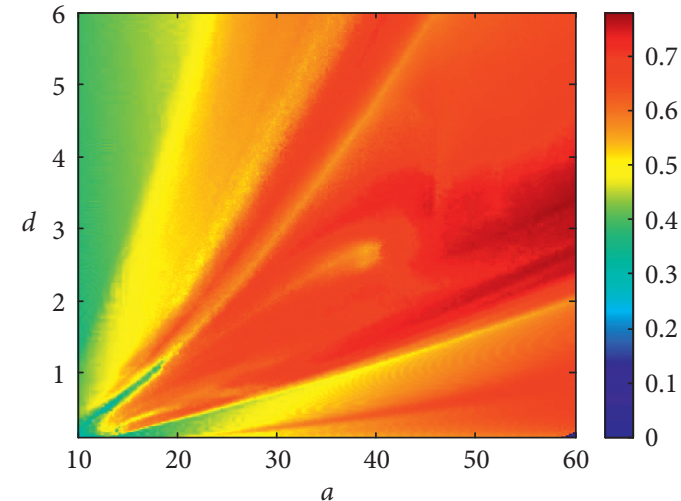

(b)

FIgURE 11: Complexity with varying parameters $a$ and $d$. (a) C0 complexity. (b) SE complexity.

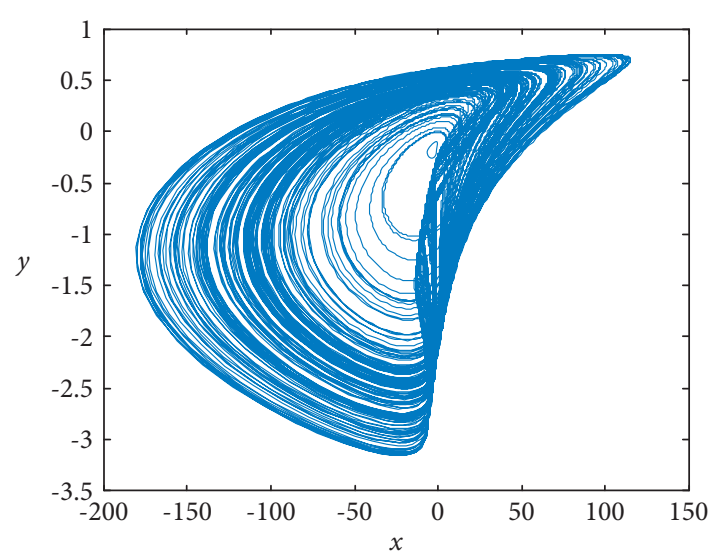

(a)

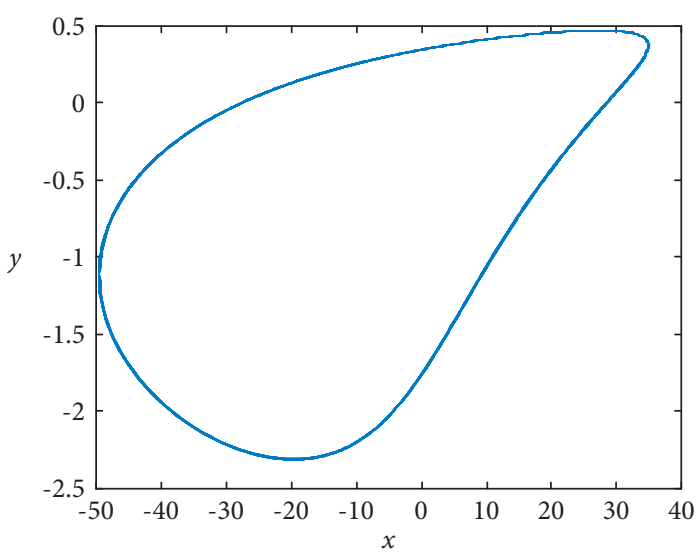

(b)

Figure 12: The phase diagram of the system. (a) $a=55.17, d=3.07$. (b) $a=11.33, d=3.05$.

recognition, and other processing, in order to get in line with people's needs of the signal form.

Because the chaotic system is easily affected by external disturbance when it is implemented by the analog circuit, it is hard to control the relevant characteristic conditions accurately in the real circuit. The DSP simulation chip used in this paper is F28335. In this section, the system will be simulated by the DSP, and the chaotic phenomenon displayed on the DSP platform will be more stable. 


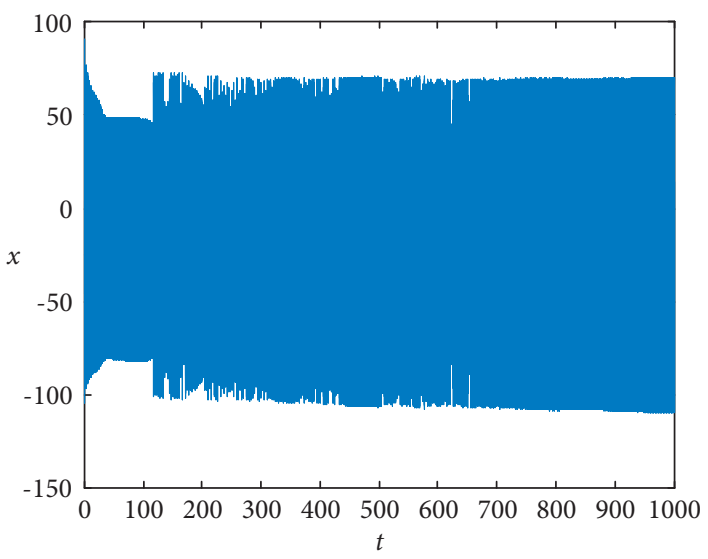

Figure 13: Timing diagram of the chaotic system with time $(0,1000)$.

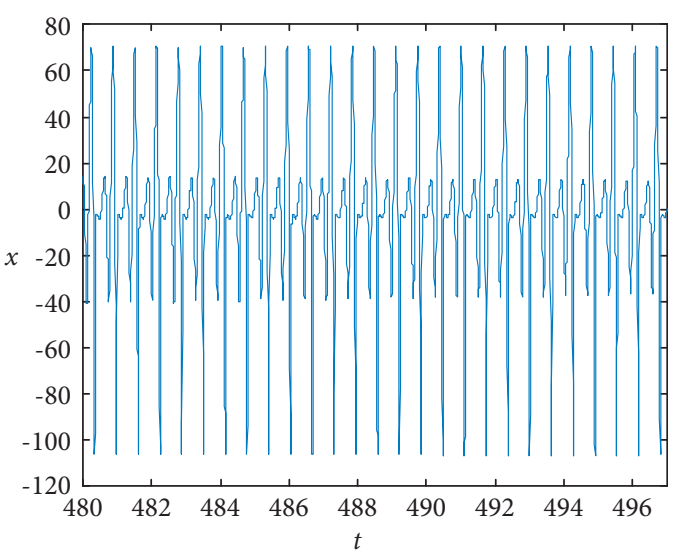

(a)

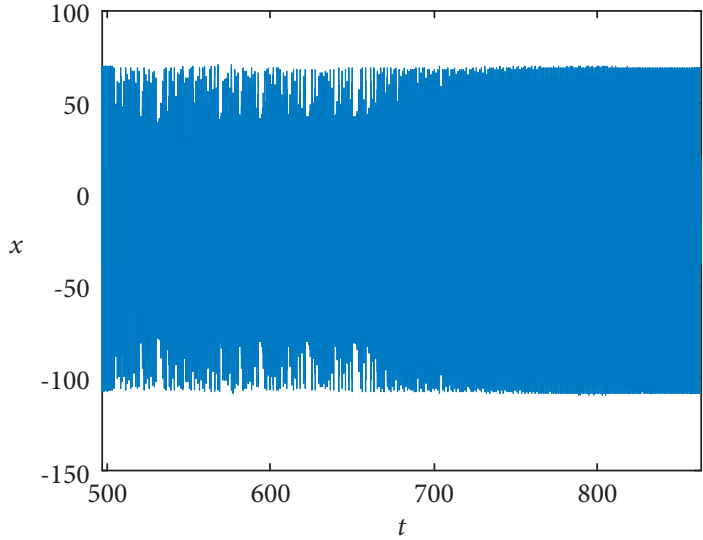

(b)

FIgURE 14: Timing diagram of the chaotic system. (a) $t \in(480,497)$. (b) $t \in(497,836)$.

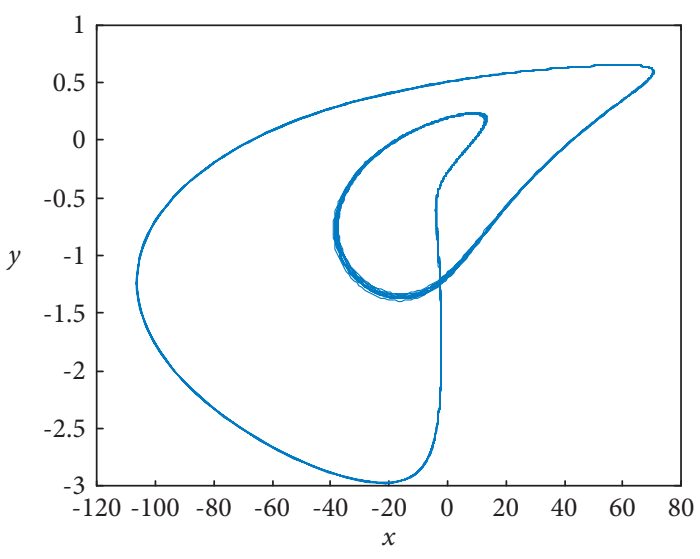

(a)

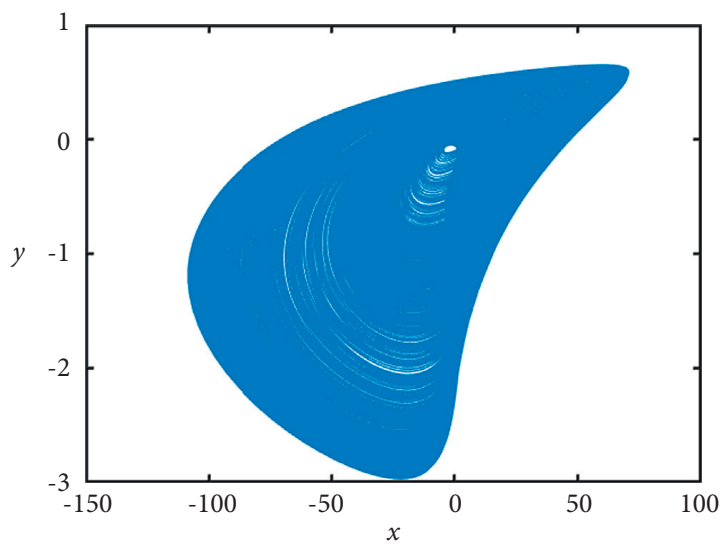

(b)

FIgURE 15: The phase diagram of the chaotic attractor. (a) $t \in(480,497)$. (b) $t \in(497,836)$.

In order to make the oscilloscope better capture images, we need a D/A converter to convert the sequence produced by the DSP into an analog sequence. The output sequence is then displayed on an oscilloscope to form an image. Their relationship is shown in Figure 17.
In order to realize the circuit on DSP, the system should be discretized first. Therefore, we use the fourthorder Runge-Kutta method to discretize the continuous chaotic system and transform it into discrete chaotic sequence. 


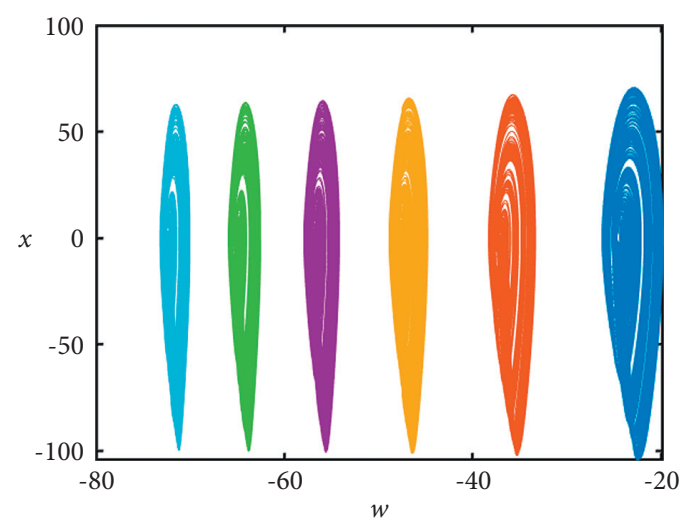

(a)

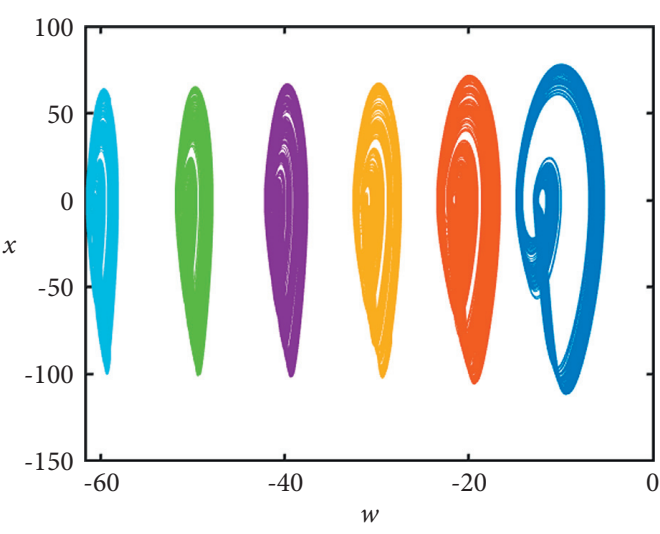

(b)

FiguRe 16: Coexisting attractors with different $z_{0}$ and $w_{0}$.

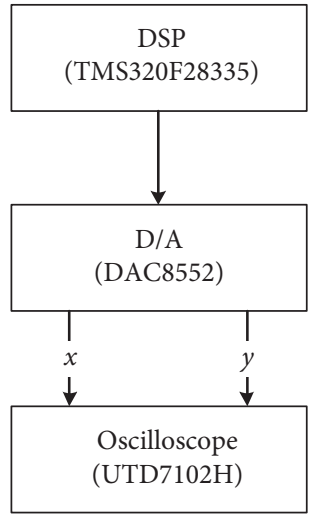

Figure 17: The flow chart.

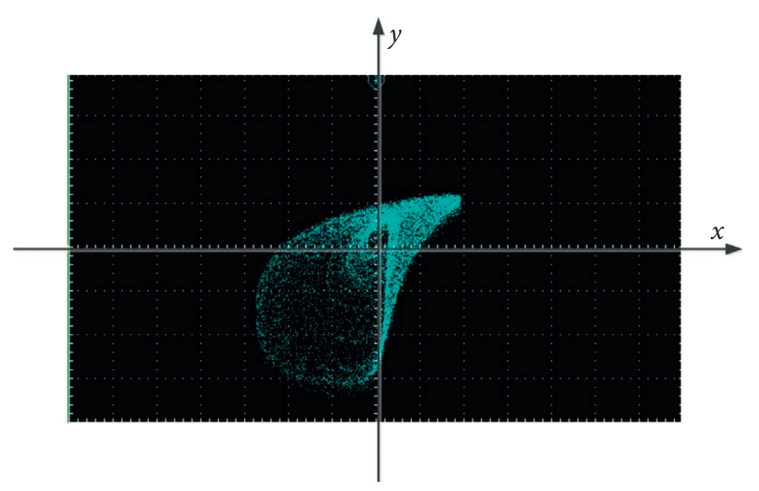

(a)

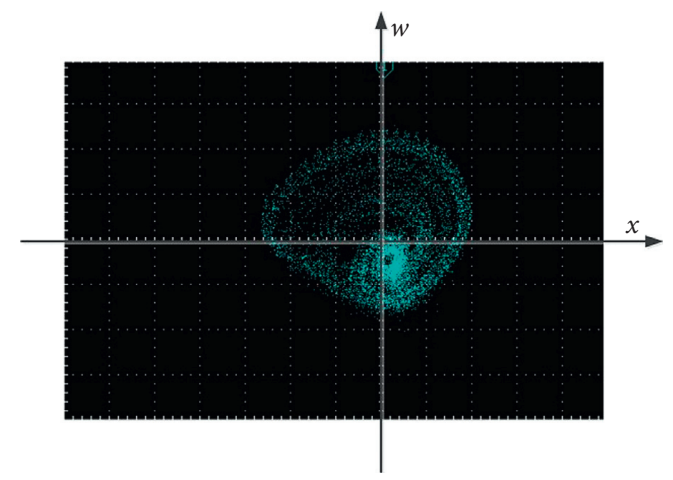

(b)

FIgURE 18: Experimental results of DSP implementation. (a) $x-y$ plane. (b) $x-w$ plane. 


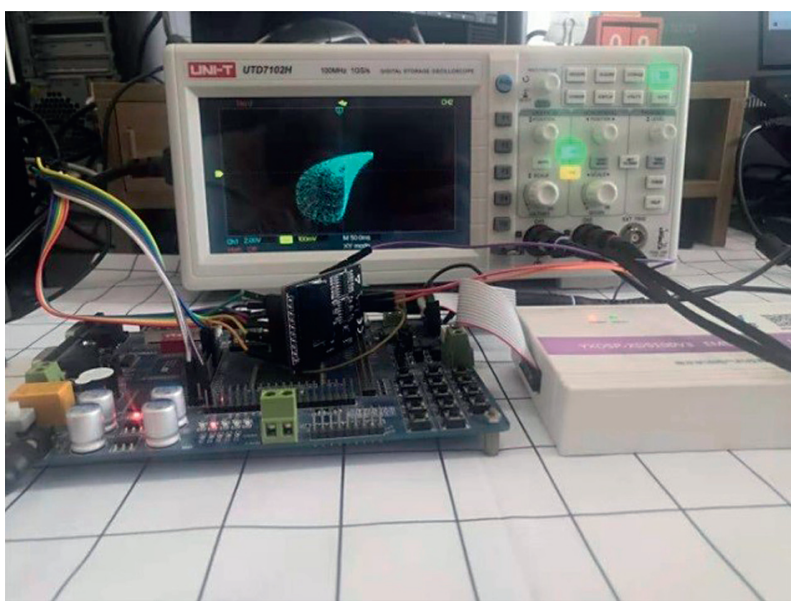

FIGURE 19: Experimental platform for DSP implementation.

Let the parameters be $a=30, b=18, d=2, c=100, e=0.2$, and $g=-0.017$, and set the initial condition as $(4,50,1,24$, -23). The image as shown in Figure 18 can be obtained on DSP. The phase diagram realized by DSP is basically consistent with the phase diagram obtained by numerical simulation.

The hardware types such as F28335 chip and oscilloscope used for DSP simulation of the system are shown in Figure 19.

\section{Conclusions}

In this paper, a chaotic oscillator consisting of memristor, meminductor, and memcapacitor in series is designed and the stability of the system is analyzed. By changing the parameters of the system, the dynamic characteristics of the system are varied. By analyzing the LEs, bifurcation diagram, and complexity of the system, we find that the system is a hyperchaotic system and the dynamic behavior of the system shows high complexity and sensitivity with the change of system parameters. The special dynamical phenomena such as coexistence of attractors and state transition are found in chaotic systems, and reasonable explanations are provided for these phenomena. Finally, the circuit is implemented on DSP and the phase diagram obtained by DSP is basically consistent with that obtained by software simulation. Because of its abundant dynamical behavior, this simple chaotic circuit has a broad application prospect in image encryption and chaotic secure communication.

\section{Data Availability}

The data used to support the findings of this study are included within the article.

\section{Conflicts of Interest}

The authors declare no conflicts of interest.

\section{Acknowledgments}

This work was supported by the National Natural Science Foundation of China (Grant nos. 62061014 and 61773010),
Natural Science Foundation of Liaoning Province (Grant no. 2020-MS-274), and Basic Scientific Research Projects of Colleges and Universities of Liaoning Province (Grant no. J202148).

\section{References}

[1] H. Poincaré, "Review of hilbert's foundations of geometry," Bulletin of the American Mathematical Society, vol. 10, no. 1903, pp. 1-23, 1903.

[2] E. N. Lorenz, "Deterministic nonperiodic flow," Journal of the Atmospheric Sciences, vol. 20, no. 2, pp. 130-141, 2004.

[3] D. Ruelle and F. Takens, "On the nature of turbulence," Communications in Mathematical Physics, vol. 20, no. 3, pp. 167-192, 1971.

[4] L. O. Chua, The Genesis of Chua's Circuit, Electronics Research Laboratory, College of Engineering, University of California, Berkeley, CA, USA, 1992.

[5] T. Liu, H. Yan, S. Banerjee, and J. Mou, "A fractional-order chaotic system with hidden attractor and self-excited attractor and its DSP implementation," Chaos, Solitons \& Fractals, vol. 145, p. 110791, 2021.

[6] T. Liu, S. Banerjee, H. Yan, and J. Mou, "Dynamical analysis of the improper fractional-order 2D-SCLMM and its DSP implementation," The European Physical Journal Plus, vol. 136, no. 5, p. 506, 2021.

[7] C. Ma, J. Mou, P. Li, and L. Tianming, "Dynamic analysis of a new two-dimensional map in three forms: integer-order, fractional-order and improper fractional-order," The European Physical Journal - Special Topics, vol. 230, pp. 1945-1957, 2021.

[8] C. Ma, J. Mou, L. Xiong, S. Banerjee, T. Liu, and X. Han, "Dynamical analysis of a new chaotic system: asymmetric multistability, offset boosting control and circuit realization," Nonlinear Dynamics, vol. 103, no. 3, pp. 2867-2880, 2021.

[9] Y. Peng, S. He, and K. Sun, "Parameter identification for discrete memristive chaotic map using adaptive differential evolution algorithm," Nonlinear Dynamics, pp. 1-13, 2021.

[10] J. Liu, G. Chen, and X. Zhao, "Generalized synchronization and parameters identification of different-dimensional chaotic systems in the complex field," Fractals, vol. 29, no. 4, pp. 2150081-2150824, 2021. 
[11] G. Peng, Y. Jiang, and C. Fang, "Generalized projective synchronization of fractional order chaotic systems," Phys.rev.e, vol. 387, no. 14, pp. 3738-3746, 2003.

[12] J. Ruan, K. Sun, J. Mou, and H. shaobo, "Fractional-order simplest memristor-based chaotic circuit with new derivative," The European Physical Journal Plus, vol. 133, no. 1, pp. 1-12, 2018.

[13] S. He, K. Sun, and H. Wang, "Dynamics and synchronization of conformable fractional-order hyperchaotic systems using the Homotopy analysis method," Communications in Nonlinear Science and Numerical Simulation, vol. 73, pp. 146-164, 2019.

[14] X. Wang, J. Yu, C. Jin, H. H. C. Iu, and S. Yu, "Chaotic oscillator based on memcapacitor and meminductor," Nonlinear Dynamics, vol. 96, no. 1, pp. 161-173, 2019.

[15] Z. Takran, M. Saba, U. E. Ayten, and S. Herman, "A new universal mutator circuit for memcapacitor and meminductor elements," AEU - International Journal of Electronics and Communications, vol. 119, Article ID 153180, 2020.

[16] B. Bao, L. Xu, N. Wang, H. Bao, Q. Xu, and M. Chen, "Thirdorder RLCM-four-elements-based chaotic circuit and its coexisting bubbles," AEU - International Journal of Electronics and Communications, vol. 94, pp. 26-35, 2018.

[17] T. Prodromakis, B. P. Peh, C. Papavassiliou, and C. Toumazou, "A vmemristor model with nonlinear dopant kinetics," IEEE Transactions on Electron Devices, vol. 58, no. 9, pp. 3099-3105, 2011.

[18] L. Chua, "Memristor-the missing circuit element," IEEE Transactions on Circuit Theory, vol. 18, no. 5, pp. 507-519, 1971.

[19] L. O. Chua and S. M. Sung Mo Kang, "Memristive devices and systems," Proceedings of the IEEE, vol. 64, no. 2, pp. 209-223, 1976.

[20] D. B. Strukov, G. S. Snider, D. R. Stewart, and R. S. Williams, "The missing memristor found," Nature, vol. 453, no. 7191, pp. 80-83, 2008.

[21] J. M. Tour and T. He, “The fourth element," Nature, vol. 453, no. 7191, pp. 42-43, 2008.

[22] C. Moreno, C. Munuera, X. Obradors, and C. Ocal, "The memory effect of nanoscale memristors investigated by conducting scanning probe microscopy methods," Beilstein Journal of Nanotechnology, vol. 3, no. 1, pp. 722-730, 2012.

[23] A. Williamson, L. Schumann, L. Hiller et al., "Synaptic behavior and STDP of asymmetric nanoscale memristors in biohybrid systems," Nanoscale, vol. 5, no. 16, pp. 7297-7303, 2013.

[24] M. Peterlechner, T. Waitz, and H. P. Karnthaler, "Nanoscale amorphization of severely deformed $\mathrm{NiTi}$ shape memory alloys," Scripta Materialia, vol. 60, no. 12, pp. 1137-1140, 2009.

[25] S. Vaidyanathan, V. T. Pham, C. K. Volos, and T. P. Le, “A memristor-based hyperchaotic system with hidden attractors: dynamics, synchronization and circuital emulating," Journal of Engineering ScienceљTechnology Review, vol. 8, no. 2, pp. 205-214, 2014.

[26] S. Vaidyanathan, A. T. Azar, A. Akgul, C. H. Lien, S. Kacar, and U. Cavusoglu, "A memristor-based system with hidden hyperchaotic attractors, its circuit design, synchronisation via integral sliding mode control and an application to voice encryption," International Journal of Automation and Control, vol. 13, no. 6, p. 644, 2019.

[27] M. Di Ventra, Y. V. Pershin, and L. O. Chua, "Circuit elements with memory: memristors, memcapacitors, and meminductors," Proceedings of the IEEE, vol. 97, no. 10, pp. 1717-1724, 2009.

[28] Z. Y. ZhenYu Yin, H. Heng Tian, G. H. GuanHua Chen, and L. O. Chua, "What are memristor, memcapacitor, and meminductor," IEEE Transactions on Circuits and Systems II: Express Briefs, vol. 62, no. 4, pp. 402-406, 2015.

[29] X. Ma, J. Mou, J. Liu, C. Ma, F. Yang, and X. Zhao, "A novel simple chaotic circuit based on memristor-memcapacitor," Nonlinear Dynamics, vol. 100, no. 3, pp. 2859-2876, 2020.

[30] Z. Hu, Y. Li, J. Li, and J. Yu, "Chaotic oscillator based on voltage-controlled memcapacitor," in Proceedings of the International Conference on Communications, July 2010.

[31] M. S. Feali, A. Ahmadi, and M. Hayati, "Implementation of adaptive neuron based on memristor and memcapacitor emulators," Neurocomputing, vol. 309, no. OCT.2, pp. 157-167, 2018.

[32] F. Min, Y. Cheng, L. Lu, and X. Li, "Extreme multistability and as oscillator with two flux-controlled memristors," International Journal of Bifurcation and Chaos, vol. 31, no. 11, Article ID 2150167, 2021.

[33] Q. Lai, Z. Wan, A. Akgul, O. F. Boyraz, and M. Z. Yildiz, "Design and implementation of a new memristive chaotic system with application in touchless fingerprint encryption," Chinese Journal of Physics, vol. 67, pp. 615-630, 2020.

[34] S. Mobayen, A. Fekih, S. Vaidyanathan, and A. Sambas, "Chameleon chaotic systems with quadratic nonlinearities: an adaptive finite-time sliding mode control approach and circuit simulation," IEEE Access, vol. 9, pp. 64558-64573, 2021.

[35] M. Itoh and L. O. Chua, "Memristor oscillators," International Journal of Bifurcation and Chaos, vol. 18, no. 11, pp. 31833206, 2008.

[36] M. Bharathwaj and L. O. Chua, "Simplest chaotic circuit," International Journal of Bifurcation \& Chaos, vol. 20, no. 5, pp. 1567-1580, 2012.

[37] B. Xu, G. Wang, and Y. Shen, "A simple meminductor-based chaotic system with complicated dynamics," Nonlinear Dynamics, vol. 88, no. 3, pp. 2071-2089, 2017.

[38] F. Yuan and Y. Li, "A chaotic circuit constructed by a memristor, a memcapacitor and a meminductor," Chaos: An Interdisciplinary Journal of Nonlinear Science, vol. 29, no. 10, Article ID 101101, 2019.

[39] J. Secco, M. Poggio, and F. Corinto, "Supervised neural networks with memristor binary synapses," International Journal of Circuit Theory and Applications, vol. 46, no. 1, pp. 221-233, 2018.

[40] M. Laiho and E. Lehtonen, "Cellular nanoscale network cell with memristors for local implication logic and synapses," in Proceedings of the IEEE International Symposium on Circuits \& Systems, pp. 2051-2054, IEEE, Paris, France, May 2010.

[41] G. Indiveri, B. Linares-Barranco, R. Legenstein, G. Deligeorgis, and T. Prodromakis, "Integration of nanoscale memristor synapses in neuromorphic computing architectures," Nanotechnology, vol. 24, no. 38, Article ID 384010, 2013.

[42] R. Naous, M. AlShedivat, E. Neftci, G. Cauwenberghs, and K. N. Salama, "Memristor-based neural networks: synaptic versus neuronal stochasticity," AIP Advances, vol. 6, no. 11, Article ID 111304, 2016.

[43] C. Chen, H. Bao, M. Chen, Q. Xu, and B. Bao, "Non-ideal memristor synapse-coupled bi-neuron Hopfield neural network: numerical simulations and breadboard experiments," $A E U$ - International Journal of Electronics and Communications, vol. 111, Article ID 152894, 2019. 
[44] H. Bao, Y. Zhang, W. Liu, and B. Bao, "Memristor synapsecoupled memristive neuron network: synchronization transition and occurrence of chimera," Nonlinear Dynamics, vol. 100, no. 1, pp. 937-950, 2020.

[45] F. Yang, J. Mou, C. Ma, and Y. Cao, "Dynamic analysis of an improper fractional-order laser chaotic system and its image encryption application," Optics and Lasers in Engineering, vol. 129, no. 2020, Article ID 106031, 2020.

[46] X. Li, J. Mou, L. Xiong, Z. Wang, and J. Xu, "Fractional-order double-ring erbium-doped fiber laser chaotic system and its application on image encryption," Optics \& Laser Technology, vol. 140, no. 2021, Article ID 107074, 2021.

[47] Y. Xian and X. Wang, "Fractal sorting matrix and its application on chaotic image encryption," Information Sciences, vol. 547, pp. 1154-1169, 2021.

[48] M. Wang, X. Wang, Y. Zhang, S. Zhou, T. Zhao, and N. Yao, "A novel chaotic system and its application in a color image cryptosystem," Optics and Lasers in Engineering, vol. 121, pp. 479-494, 2019.

[49] H. Wu, H. Zhu, and G. Ye, "Public key image encryption algorithm based on pixel information and random number insertion," Physica Scripta, vol. 96, no. 10, Article ID 105202, 2021.

[50] S. Zhang, J. Zheng, X. Wang, Z. Zeng, and S. He, "Initial offset boosting coexisting attractors in memristive multi-doublescroll Hopfield neural network," Nonlinear Dynamics, vol. 102, no. 4, pp. 2821-2841, 2020.

[51] X. An and S. Qiao, "The hidden, period-adding, mixed-mode oscillations and control in a HR neuron under electromagnetic induction," Chaos, Solitons \& Fractals, vol. 143, Article ID 110587, 2021.

[52] S. Qiao and A. Xin-Lei, "Dynamic expression of a HR neuron model under an electric field," International Journal of Modern Physics B, vol. 35, no. 2, Article ID 2150024, 2021.

[53] A. Xin-Lei, S. Qiao, and L. Zhang, "Dynamic response and control of neuros based on electromagnetic field theory," Acta Physica Sinica, vol. 70, no. 5, Article ID 050501, 2021.

[54] Yu Fei, H. Shen, Z. Zhang, Y. Huang, S. Cai, and S. Du, "Dynamics analysis, hardware implementation and engineering applications of novel multi-style attractors in a neural network under electromagnetic radiation, Chaos," Solitons \& Fractals, vol. 152, Article ID 111350, 2021.

[55] Y. Fei, H. Shen, Z. Zhang, Y. Huang, S. Cai, and S. Du, “A new multi-scroll Chua's circuit with composite hyperbolic tangent-cubic nonlinearity: complex dynamics, Hardware implementation and Image encryption application," Integration, vol. 81, pp. 71-83, 2021.

[56] G. Peng and F. Min, "Multistability analysis, circuit implementations and application in image encryption of a novel memristive chaotic circuit," Nonlinear Dynamics, vol. 90, no. 3, pp. 1607-1625, 2017.

[57] L. Chai, J. Liu, and G. R. Chen, "Dynamics and synchronization of a complex-valued star network," Science China Technological Sciences, vol. 64, pp. 2729-2743, 2021.

[58] A. Sambas, S. Vaidyanathan, E. Tlelo-Cuautle et al., "A 3-D multi-stable system with a peanut-shaped equilibrium curve: circuit design, FPGA realization, and an application to image encryption," IEEE Access, vol. 8, pp. 137116-137132, 2020.

[59] H. Wang, X. Wang, C. Li, and C. Ling, "SPICE mutator model for transforming memristor into meminductor," Abstract and Applied Analysis, Article ID 281675, 5 pages, 2013.

[60] Q. Zhao, C. Wang, and X. Zhang, "A universal emulator for memristor, memcapacitor, and meminductor and its chaotic circuit," Chaos: An Interdisciplinary Journal of Nonlinear Science, vol. 29, no. 1, Article ID 013141, 2019.

[61] F. Yuan, Y. Deng, Y. Li, and G. Wang, "The amplitude, frequency and parameter space boosting in a memristormeminductor-based circuit," Nonlinear Dynamics, vol. 96, no. 1, pp. 389-405, 2019.

[62] B. Han, W. Liu, A. Hu, H. Bao, W. Liu, and A. Hu, "Coexisting multiple firing patterns in two adjacent neurons coupled by memristive electromagnetic induction," Nonlinear Dynamics, vol. 95, pp. 43-56, 2019.

[63] S. He, K. Sun, H. Wang, X. Mei, and Y. Sun, "Generalized synchronization of fractional-order hyperchaotic systems and its DSP implementation," Nonlinear Dynamics, vol. 92, no. 1, pp. 85-96, 2018.

[64] S. He, K. Sun, and H. Wang, "Complexity analysis and DSP implementation of the fractional-order Lorenz hyperchaotic system," Entropy, vol. 17, no. 12, pp. 8299-8311, 2015.

[65] S. Dai, K. Sun, W. Ai, and Y. Peng, "Novel discrete chaotic system via fractal transformation and its DSP implementation," Modern Physics Letters B, vol. 34, no. 1, Article ID 2050429, 2020.

[66] A. Sambas, S. Vaidyanathan, T. Bonny et al., "Mathematical model and FPGA realization of a multi-stable chaotic dynamical system with a closed butterfly-like curve of equilibrium points," Applied Sciences, vol. 11, no. 2, p. 788, 2021.

[67] A. Sambas, S. Vaidyanathan, S. Zhang, Y. Zeng, M. A. Mohamed, and M. Mamat, "A new double-wing chaotic system with coexisting attractors and line equilibrium: bifurcation analysis and electronic circuit simulation," IEEE Access, vol. 7, pp. 115454-115462, 2019.

[68] Q. Lai, "A unified chaotic system with various coexisting attractors," International Journal of Bifurcation and Chaos, vol. 31, no. 1, Article ID 2150013, 2021.

[69] Q. Lai, Z. Wan, L. K. Kengne, P. D. Kamdem Kuate, and C. Chen, "Two-memristor-based chaotic system with infinite coexisting attractors," IEEE Transactions on Circuits and Systems II: Express Briefs, vol. 68, no. 6, pp. 2197-2201, 2021.

[70] Q. Lai, Z. Wan, and P. D. Kamdem Kuate, "Modelling and circuit realisation of a new no-equilibrium chaotic system with hidden attractor and coexisting attractors," Electronics Letters, vol. 56, no. 20, pp. 1044-1046, 2020. 